\title{
Sauna: uma atividade saudável?
}

\author{
Marcos Aurelio Brazão de Oliveira ${ }^{1}$
}

\section{INTRO DUÇÃO}

A sauna ou o "banho de sauna" é uma prática relativamente comum em clubes recreativos, possui bom número de adeptos em nosso meio e, não raro, o médico é consultado sobre os riscos e benefícios desse tipo de atividade, seja para indivíduos aparentemente sadios, seja para os portadores de determinadas patologias, com ou sem uso de medicamentos. Por essas razões, iremos abordar alguns aspectos importantes relacionados com aconselhamento médico para os praticantes desse tipo de atividade.

\section{RESPOSTAS GERAIS DO ORGANISMO AO CALOR}

Para se adaptar às grandes variações de temperatura ambiente o organismo humano lança mão de um mecanismo de termorregulação altamente eficiente cujo centro de controle se encontra no hipotálamo(1). Esse centro de controle de temperatura funciona como um termostato que é ajustado a um ponto crítico para cima e outro para baixo. No caso da exposição ao calor, quando a temperatura do sangue se encontra acima do ponto crítico, o hipotálamo envia uma mensagem aos vasos cutâneos com o objetivo de provocar uma vasodilatação que irá facilitar a perda de calor. Haverá, então, duas possibilidades: a) se a temperatura do ar for inferior à da pele, os mecanismos de irradiação, condução e convecção poderão ser suficientes para "ajustar" a temperatura do corpo; b) se a temperatura do ar for mais elevada do que a da pele, os dispositivos citados serão insuficientes para eliminar o calor excedente e ocorrerá, então, a evaporação do suor que é produzido por cerca de 2,5 milhões de glândulas sudoríparas(17) e cuja ativação é feita através da via colinérgica e dos neurônios pós-ganglionares.

Bligh e J ohnson(2) chamaram de "aclimatação" ao conjunto de alterações fisiológicas que sofre o organismo quando submetido a um stress calórico induzido artificialmente.

\section{DIFERENÇAS DE RESPOSTA DE EXPOSIÇÃO AO CALOR}

- Na mulher

Os fenômenos adaptativos descritos anteriormente apresentam certas particularidades em relação a sexo e idade.

1. Pós-graduado em Med. Desp. pela UFRJ ; Cardiolog. e Méd. Desp. do Serv. Social do Com. (SESC-RJ)); Pres. da Soc. de Med. Desp. do Rio de J aneiro; Editor Científico do "J ornal de Medicina do Exercício".
Está bem demonstrado que a mulher é mais vulnerável ao calor e os fatores que concorreriam para isto seriam: menor volume sanguíneo e maior área de superfície em relação à massa corporal, o que faz com que a quantidade de sangue que a pele recebe por unidade de superfície seja menor, 0 que dificulta a troca de calor. O utro fator seria a existência de temperaturas centrais ligeiramente superiores na mulher em sua fase luteínica do ciclo hormonal. Todos esses fatores contribuem para que a mulher seja mais sensível ao calor do que o homem. Corroborando com essa assertiva, J ezova et al. ${ }^{(7)}$, em trabalho publicado em março de 1994, investigando em oito mulheres e oito homens (todos sadios) a resposta neurendócrina durante e após o banho de sauna a $80^{\circ} \mathrm{C}$ através da análise dos níveis de cortisol, ACTH, adrenalina e noradrenalina, concluíram que a hipertermia induzida pela sauna provocou ativação neurendócrina mais pronunciada nas mulheres do que nos homens.

\section{- No idoso}

A resposta do organismo ao calor no idoso não difere muito dos mais jovens, apesar de ele possuir uma reserva circulatória menor; entretanto, é importante lembrar que comumente o idoso faz uso de algum tipo de medicamento que pode modificar sua resposta ao calor.

\section{- Na criança}

Segundo Becerro(1), nas crianças a resposta do organismo nos ambientes quentes difere bastante da resposta dos adultos. Isto é explicado pelo fato de elas possuírem uma área de superfície corporal em relação à massa muscular $35 \%$ maior que a dos adultos, razão pela qual o corpo ganha temperatura com maior rapidez quando exposto ao calor. Finalmente, apesar de as crianças possuírem maior número de glândulas sudoríparas por $\mathrm{cm}^{2}$ de pele, a quantidade de suor produzido por cada uma delas é 2,5 vezes inferior à dos adultos.

\section{RESPOSTAS CARDIOVASCULARES AO BANHO DE SAUNA}

Existem poucos estudos controlados do ponto de vista médico e fisiológico disponíveis na literatura em relação ao banho de sauna. Um dos trabalhos clássicos é o de Sohar et al. (12), que estudaram 33 homens e 27 mulheres cujas idades variaram de 18 a 63 anos. Esses voluntários entraram no banho de sauna após 20 minutos de repouso prévio e permaneceram por 20 minutos ou até referirem desconforto. Foram analisados peso, altura, temperaturas retal e cutâ- 
nea, pressão arterial, freqüências cardíaca e respiratória e eletrocardiograma 20 minutos antes, durante e 20 minutos após deixarem a sauna. As marcadas alterações fisiológicas surgiram nos primeiros minutos sem nenhum pródromo. A os 20 minutos a FC média atingiu $143 \pm 25$ bpm (em $32 \%$ dos indivíduos ultrapassou $160 \mathrm{bpm}$ ); a média da pressão sistólica ficou em torno de 130,5 $\pm 26,6 \mathrm{mmH} \mathrm{g}$ (maior que $160 \mathrm{mmH} \mathrm{g} \mathrm{em} 17 \%$ dos casos) e a média da pressão diastólica chegou a $66,6 \pm 15,9 \mathrm{mmH}$ g (menor que $50 \mathrm{mmH} \mathrm{g} \mathrm{em}$ $17 \%$ dos casos). Três indivíduos tiveram síncope e um desenvolveu angina pectoris. Não houve queda da pressão arterial nos indivíduos que apresentavam hipertensão arterial prévia. Em 12 indivíduos (20\%) foi observada no ECG uma depressão da onda T e em dez indivíduos (16,6\%) uma depressão significativa do segmento ST. Essas alterações eletrocardiográficas foram atribuídas ao stress cardiovascular induzido pelo calor. Alterações semelhantes já haviam sido descritas por Taggart et al.(13).

Os autores concluem que o banho de sauna pode se constituir num elemento de risco para a saúde e que os efeitos indesejáveis provocados por ela podem surgir subitamente sem sinais prodrômicos.

Em outro trabalho, Trezza et al.(16) estudaram 46 adultos do sexo masculino antes e após o banho de sauna seca (17 indivíduos) e úmida (29 indivíduos), os quais foram analisados como um grupo único, por não terem apresentado diferenças nas variáveis estudadas nos dois tipos de sauna. Nesse estudo não houve diferença significativa da freqüência respiratória. A FC passou de 97 para $108 \mathrm{bpm}$ na posição ortostática. A pós o banho de sauna, foi observada hipotensão postural, com diminuição mais intensa da P. diastólica. Dois indivíduos apresentaram lipotimia quando adotaram a posição de pé (um deles com perda da consciência). No grupo havia dois hipertensos cujas respostas foram totalmente distintas: um deles mostrou hipotensão postural $(180 \times 110$ para $130 \times 80 \mathrm{mmHg}$ ) e o outro revelou exacerbação. Diferentemente do trabalho de Sohar, o ECG não mudou significativamente e não foram constatadas alterações da repolarização ventricular após a sauna.

A o final do trabalho os autores alertam para os riscos de hipotensão arterial em pacientes com uso de coronariodilatadores ou anti-hipertensivos.

Luurila(11), por sua vez (em artigo citado por Trezza), advertiu para o risco de picos hipertensivos importantes provocados pelo banho muito frio logo após a sauna, já tendo registrado cifras tensionais de até $300 \mathrm{mmHg}$ de $P$. sistólica e até $200 \mathrm{mmH}$ g de diastólica.

\section{SAUNA E FUNÇÃO RESPIRATÓRIA}

Kiss et al.(9), analisando os efeitos do banho de sauna sobre a capacidade de difusão, função pulmonar, débito e índice cardíacos, concluíram que o calor do banho de sauna provocou discreto, mas não significativo, aumento da capacidade de difusão ( $p=0,239)$ e nenhuma alteração nos outros parâmetros da função pulmonar. Os autores encontraram, também, discreto, mas não significativo, aumento do débito cardíaco $(p=0,2455)$ e do índice cardíaco $(p=$ $0,2719)$.

\section{SAUNA E INSUFICIÊNCIA CORONARIANA}

Luurila et al. ${ }^{(11)}$, estudando a resposta cardiovascular em 37 pacientes com história de infarto do miocárdio que, tardiamente, voltaram a freqüentar sauna, encontraram baixa incidência de alterações cardiocirculatórias, alterações essas bem menores do que as provocadas pelo teste ergométrico nesses mesmos pacientes, tanto no que diz respeito às anormalidades do segmento ST tipo isquêmicas, quanto em relação à incidência de arritmias cardíacas. Esses resultados diferem daqueles encontrados por outros autores, que acreditam que a taquicardia e o aumento do débito cardíaco nesses pacientes seriam fatores predisponentes para 0 aparecimento de incidência maior de alterações clínicas e/ ou eletrocardiográficas.

\section{SAUNA E INSUFICIÊNCIA CARDÍACA}

Em artigos publicados em maio/94 e junho/95, Tei et al. ${ }^{(14,15)}$, estudaram os efeitos hemodinâmicos da vasodilatação térmica provocada pelo banho de sauna (BS) e banho de água quente (BAQ) em 34 pacientes portadores de insuficiência cardíaca crônica. A média de idade do grupo foi 58 \pm 14 anos e a fração de ejeção $25 \pm 9 \%$. A pós a colocação de um cateter Swan-Ganz na veia jugular direita, o paciente era submetido a um BAQ por 10 minutos $a 1^{\circ} \mathrm{C}$ ou BS por 15 minutos a $60^{\circ} \mathrm{C}$. Foram monitorizados a pressão arterial, o eletrocardiograma, o ecodoppler e as pressões intracardíacas. O s resultados mostraram aumento da fração de ejeção tanto no BS quanto no BAQ; a pressão sistólica não revelou alterações significativas e a pressão diastólica diminuiu significativamente durante o BS. As médias das pressões da artéria pulmonar, capilar pulmonar e do átrio direito aumentaram significativamente durante $0 \mathrm{BAQ}$, mas não no BS. Os autores acreditam que as melhoras hemodinâmicas após o BS e BAQ se deveram à redução do preload e after-load e propõem a utilização térmica, segundo eles com pouco risco, como forma de terapia não farmacológica em portadores de insuficiência cardíaca crônica, desde que utilizada de maneira adequada.

\section{SAUNA E DIABETES MELITO}

Vários trabalhos têm demonstrado que existe aumento da absorção de insulina nos pacientes diabéticos insulinodependentes que freqüentam sauna. Num desses trabalhos, Koivisto(10), por exemplo, analisando oito pacientes que se 
utilizavam de insulina por via subcutânea e que se submeteram a um banho de sauna duas vezes por 25 minutos, a uma temperatura de $85^{\circ} \mathrm{C}$, constatou, através de um marcador radioativo (iodo 125), que houve aumento na absorção de insulina em cerca de $110 \%$, em comparação com a sala com temperatura normal. A pós a sauna, as concentrações de glicose foram 3,0 e $3,3 \mathrm{~mm} / \mathrm{I}(54,1-59,5 \mathrm{mg} \%)$ mais baixas do que no dia-controle $(p<0,05)$. A queda da glicemia foi proporcional à absorção de insulina $(r=0,30 ; p<0,01)$.

0 autor adverte que esse efeito hipoglicemiante que a sauna exerce sobre os diabéticos insulino-dependentes pode ser minimizado com a diminuição da dose de insulina nos dias em que os mesmos forem se submeter a esse stress térmico.

\section{SAUNA E PORTADORES DE MARCAPASSO CARDÍACO}

Em recente artigo (fevereiro de 1997) sobre "O rientações a respeito de interferências sobre marcapassos artificiais", Gauch et al. ${ }^{(3)}$ explicam que, apesar da sofisticação progressiva dos marcapassos que envolvem os sensores, sua adequação fisiológica para as diversas solicitações hemodinâmicas ainda é incompleta. Os autores concluem afirmando que, embora não interfiram diretamente nos marcapassos, situações que provocam vasodilatação importante, como saunas prolongadas, podem, dependendo do tipo e do modo de estimulação, ocasionar sintomas de baixo débito e que isso não deve ser interpretado como interferência no marcapasso e sim como uma inadequação da resposta cronotrópica frente a uma solicitação metabólica exacerbada.

\section{ACONSELHAMENTO MÉDICO PARA OS CANDIDATOS AO BANHO DE SAUNA}

Não existe consenso em relação aos riscos do banho de sauna. Alguns autores o consideram uma atividade segura. Kauppinen ${ }^{(8)}$, por exemplo, afirma em artigo recente que os banhos de sauna são muito bem tolerados e não se constituem em risco para pessoas sadias, tanto para crianças como para adultos, incluindo mulheres com gravidez em curso sem complicações. A pesar de a bibliografia não ser muito rica em trabalhos abordando os riscos do banho de sauna, os estudos desenvolvidos até o momento nos permitem tirar algumas ilações em relação ao aconselhamento médico para os freqüentadores deste tipo de atividade.

1) É conveniente uma avaliação médica de todo candidato ao banho de sauna, com particular ênfase para o apareIho cardiovascular.

2) Não é recomendável que indivíduos portadores de cardiopatia freqüentem sauna, pelo menos desacompanhados. Essa opinião emitida por Luurilla e corroborada por Trezza se justifica pelo fato de que pacientes portadores da mesma patologia podem apresentar respostas totalmente distintas frente a altas temperaturas, muitas das vezes com efeitos deletérios para o aparelho cardiovascular (pico hipertensivo, hipotensão, arritmias, alterações de ST, etc.), existindo, inclusive, casos de morte súbita durante 0 banho de sauna descritos na literatura(18).

3) Deve-se atentar para o fato de que o banho muito frio logo após a sauna pode provocar efeitos indesejáveis, como picos hipertensivos ou crises anginosas devido à abrupta vasoconstrição.

4) Em indivíduos aparentemente sadios não existem trabalhos que comprovem cientificamente algum benefício do banho de sauna(5-7,12).

5) Nos pacientes diabéticos insulino-dependentes deverá ser diminuída a dosagem da medicação nos dias em que estes forem se expor ao banho de sauna, para evitar o risco da hipoglicemia pelo aumento da absorção de insulina provocado pelo stress térmico.

6) Gumener et al.(4), estudando as respostas adaptativas de crianças ao banho de sauna, observaram que existem grandes diferenças nessas respostas em crianças da mesma idade. Esta é uma das várias razões pelas quais a sauna não parece ser uma atividade adequada para crianças.

\section{REFERÊNCIAS}

1. Becerro J F: Calor y frio. La respuesta del organismo a su acción. Arch Med Deporte 13: 47-56, 1996.

2. Bligh J, J ohnson H G: Glossary of terms for thermal physiology. J Appl Physiol 35: 941-961, 1973.

3. Gauch J RA, Halperin C, Galvão $F \circ S S$, Paula AAV, Martinelli $F \circ M$, Costa R, Pimenta J , Tarso P, Medeiros J , Brito MR, Greco OT: Orientações a respeito das interferências sobre marcapassos cardíacos. Arq Bras Cardiol 68: 1997.

4. Gumener PI, Kaisina OV, Nadezhina LG, Shumkova TV: The individual measuring of the health-promoting impact of the sauna on preschoolers. Vopr Kurortol Fizioter Lech Fiz Kult (5): 32-35, 1994.

5. Hasan JH, Karvonen MJ, Phronen P: Physiological effects of extreme heat; as studied in the Finnish sauna bath (part 1). Am J Phys Med 45: 296, 1966.

6. Hasan J H, Karvonen MJ, Phronen P: Physiological effects of extreme heat; as studied in the Finnish sauna bath (part 2). AmJ Phys Med 46: 1226, 1967.

7. J ezova $D, K$ vetnansky $R$, Vigas $M$ : Sex differences in endocrine response to hyperthermia in sauna. Acta Physiol Scand 150: 293-298, 1994.

8. Kauppinen K: Facts and fables about sauna. Ann N Y Acad Sci 813: 654-662, 1997.

9. Kiss D, Popp W, Wagner C, Swick H, Sertl K: Effects of the sauna on diffusing capacity, pulmonary function and cardiac output in healthy subjects. Respiration 61: 86-88, 1994.

10. Koivisto VA: Sauna induced acceleration in insulin absorption from subcutaneous injection site. Br Med J 280: 1411-1413, 1980.

11. Luurila J : A rrythmias and other cardiovascular responses during Finnish sauna and exercise testing in health men and postmyocardial infarction patients. Acta Med Scand Suppl: 641, 1980.

12. Sohar E, Shoenfeld J, Shapiro Y, O hry, Cabile S: Effects of exposure to Finnish sauna. Isr J Med Sci 12: 1275, 1976.

13. Taggart $P$, Parkinson $P$, Carrulhers $M:$ Cardiac response to thermal, physical and emotional stress. Br Med J 3: 71, 1972. 
14. Tei C, H orikiri Y, Park J C, J eong J W, Chang KS, Toyama Y, Tanaka N: A cute hemodynamic improvement by thermal vasodilatation in congestive heart failure. Circulation 91: 2582-2590, 1995.

15. Tei C, H orikiri Y, Park J C, J eong J W, Chang KS, Tanaka N, Toyama Y: Effects of hot water bath or sauna on patients with congestive heart failure: acute hemodynamic improvement by thermal vasodilation. J Cardiol 24: 175-183, 1994.
16. Trezza E, Bertoli Filho R, A bud Filho T: Resposta cardiocirculatória e eletrocardiográfica ao banho de sauna. Arq Bras Cardiol 46: 411-414, 1986.

17. Vander A J Sherman J H, Luciano DS: Human physiology: the mechanisms of body function, New York, McG raw-H ill, 1975.

18. Viori I: Sudden death during and after sauna bath (A bstr.), Proc. 13th Int Cong Intern Med, Helsinki, 1976, p. 285. 